\title{
Nikotinkonsum und Neurodermitis vom Late-onset-Typ
}

Die prä- und postnatale Exposition von Kindern gegenüber Tabakrauch ist mit einem erhöhten Asthma-Risiko assoziiert. Auch wenn einige Studien einen Einfluss einer (frühen) Passivrauchexposition auf die Entstehung des atopischen Ekzems zeigten, ist die Studienlage hier nicht eindeutig.

$\mathrm{n}$ der Fall-Kontroll-Studie aus Taiwan befassten sich die Autoren explizit mit dem Subtyp des atopischen Ekzems, der sich erst nach der Adoleszenz manifestiert (adult-onset atopic dermatitis). Die Fragestellung war, ob hier aktives oder passives Rauchen eine Rolle spielt.

83 Patienten mit ärztlich diagnostiziertem atopischem Ekzem vom Adultonset-Typ wurden mit 142 alters- und geschlechtgematchten Kontrollpersonen bezüglich Raucherstatus und passiver Exposition gegenüber Rauch verglichen.

Sowohl gegenwärtiges Rauchen wie auch ein Zigarettenkonsum in der Vergangenheit waren in der Regressionsanalyse signifikante Risikofaktoren für das spätere Auftreten eines atopischen Ekzems. Die Odds Ratios im Vergleich zu Individuen mit leerer Raucheranamnese betrugen 4,99 bzw. 3,6. Im Sinne eines Dosis-Wirkungs-Effekts ließ sich die Entwicklung einer atopischen Dermatitis signifikant mit der Menge der konsumierten Zigaretten korrelieren.

Darüber hinaus entwickelte sich auch bei Nichtrauchern, die als Kind in einer Wohnung mit Rauchern aufwuchsen, signifikant öfter ein atopisches Ekzem als bei Personen, die als Kind nicht Tabakrauch exponiert waren. Die entsprechende Odds Ratio für Nichtraucher, die im Alter unter 20 Jahren exponiert waren, lag bei 2,92.

Fazit: Frühe und/oder aktuelle Exposition gegenüber Zigarettenrauch kann kumulativ zur Entwicklung einer Late-onsetNeurodermitis beitragen.

Waltraud Paukstadt

Lee $\mathrm{CH}$ et al. Lifetime exposure to cigarette smoking and the development of adult-onset atopic dermatitis.

Br J Dermatol 2011; 164: 483-9

\section{Vulvogenitale Ulzerationen durch Kuhpocken}

Seit einigen Jahren wird eine Zunahme von Kuhpocken-Infektionen registriert. Diese Infektion verursacht nach Tierkontakt Läsionen, die bei Autoinokulation auch im Genitalbereich auftreten können.

K uhpocken kamen früher hauptsächlich bei Rindern vor. Ansteckungen des Menschen erfolgten hauptsächlich beim Melken („Melkerknoten“). Diese Erreger treten jetzt zunehmend bei Katzen auf. Als Wirtstiere fungieren Nagetiere, besonders Mäuse und Ratten. Die Katzen stecken sich beim Fressen infizierter Mäuse an und werden so zur Infektionsquelle für den Menschen. Auch durch Nagetiere aus Tierhandlungen können Kuhpocken übertragen werden. Ansteckungen des Menschen scheinen auch durch Hautkontakt mit kontaminierten Gegenständen wie Stacheldraht oder Beeren möglich.

Nach einer Inkubationszeit von sieben bis zwölf Tagen treten beim Menschen grippeähnliche Beschwerden auf, gefolgt von einem diffusen entzündlichen Exanthem. In der Folge kann es dann zur Ausbildung von Vesikeln kommen, die

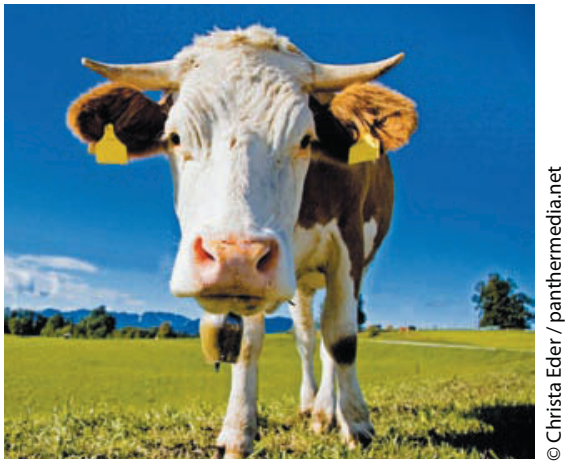

